\title{
Pratiques
}

Linguistique, littérature, didactique

\section{Peinture et littérature face aux pratiques, gouts et savoirs culturels des élèves du second degré}

Painting and literature dealing with the practices, tastes and cultural knowledge of students of the second degré

\section{Marie-Sylvie Claude}

\section{(2) OpenEdition}

\section{Journals}

Édition électronique

URL : https://journals.openedition.org/pratiques/3577

DOI : $10.4000 /$ pratiques.3577

ISSN : 2425-2042

Éditeur

Centre de recherche sur les médiations (CREM)

Référence électronique

Marie-Sylvie Claude, «Peinture et littérature face aux pratiques, gouts et savoirs culturels des élèves du second degré », Pratiques [En ligne], 175-176 | 2017, mis en ligne le 22 décembre 2017, consulté le 21 septembre 2021. URL : http://journals.openedition.org/pratiques/3577 ; DOI : https://doi.org/ 10.4000/pratiques.3577

Ce document a été généré automatiquement le 21 septembre 2021

(c) Tous droits réservés 


\section{Peinture et littérature face aux pratiques, gouts et savoirs culturels des élèves du second degré}

Painting and literature dealing with the practices, tastes and cultural knowledge of students of the second degré

\section{Marie-Sylvie Claude}

1 La réflexion proposée dans cet article s'articule sur une recherche doctorale qui tente de croiser sociologie des apprentissages et didactique pour interroger l'intégration de la lecture de la peinture dans le cours de français (Claude, 2015). Les programmes de la discipline, en France, donnent une place croissante aux arts visuels. Par exemple, dans la partie intitulée "Lecture et compréhension de l'écrit et de l'image» des nouveaux programmes de collège (MEN, 2016, p. 236 pour le cycle 4), il est question d'acquérir des "éléments d'analyse de l'image ", de "fréquenter des œuvres d'art ", d'aborder le « dialogue entre les arts ». La "visite de musée » est une des activités conseillées. Plus précisément, le travail sur l'image est préconisé comme un détour à même de faciliter l'acquisition de compétences susceptibles de bénéficier à la lecture littéraire : « les images sont l'occasion de les confronter à des procédés sémantiques proches de ceux utilisés pour les textes » (p. 240). Cette évolution des programmes pourrait s'expliquer par le fait que les élèves sont réputés plus familiers des images que des textes, comme souligné dans un rapport de l'Inspection générale, « la démarche d'analyse pour "déchiffrer" une image est plus spontanément reçue par les élèves ", de sorte que l'image peut être "un détour pédagogique opérant pour établir une écoute, une adhésion au départ refusés » (Waysbord-Loing, 2000, p. 16).

2 Les programmes incluent la peinture parmi les images ${ }^{1}$. Or, on peut douter qu'apprendre à donner sens à la peinture puisse faciliter la même opération sur la littérature, les deux arts étant très différents sur le plan sémiotique : N. Goodman (1990) désigne la peinture comme un système symbolique "dense ", qu'on ne peut décomposer en un nombre fini d'éléments, contrairement aux langages articulés. U. Eco (1992) écrit que les diverses 
combinaisons de teintes, de textures et de traits ne font pas l'objet d'un encodage de type linguistique : un tableau élabore ses propres corrélations entre composantes plastiques et contenu en même temps qu'il élabore ce contenu. Par ailleurs, on peut penser que certaines peintures, comme la peinture non figurative ou la peinture d'histoire, notamment religieuse, sont d'accès difficile pour les élèves, plus particulièrement pour ceux qui n'y ont pas été familiarisés dans leur milieu familial.

C'est pourquoi nous avons souhaité interroger, d'après des productions écrites de collégiens et de lycéens, ce détour par l'image supposé facilitateur. Nous avons obtenu de 350 élèves de troisième et de seconde de l'académie de Créteil, scolarisés dans des établissements de recrutement socialement nettement contrasté (de $60 \%$ de catégories socioprofessionnelles défavorisées à $10 \%$ ), qu'ils rédigent un commentaire d'extrait littéraire et un autre d'une reproduction picturale. Les conditions de travail étaient les mêmes pour les deux exercices et la consigne était rédigée semblablement : « écrivez dix lignes (au minimum) pour commenter (donnez quelques-unes de vos impressions) $»^{2}$. Suivait une phrase autorisant à ne pas traiter l'une de ces deux activités en expliquant pourquoi. Nous avons mené une double analyse comparative du corpus obtenu, d'une part selon les objets (pictural ou littéraire), d'autre part selon les établissements. Nous avons complété ce matériau par des entretiens postpassation avec 11 groupes de 2 à 4 élèves issus des établissements les plus défavorisés. Pour référence de ce que les élèves sont supposés apprendre, nous nous sommes appuyée sur le modèle de la lecture littéraire comme « va-et-vient dialectique » (Dufays, Gemenne \& Ledur, 2015, p. 93) entre, d'une part, la participation du lecteur (l'activité du «lu» et du «liseur» de M. Picard [1986]), le plein exercice de l'intentio lectoris (Eco, 1985), et, d'autre part, la lecture distanciée (l'activité du « lectant»), attachée à la ressaisie du matériau obtenu eu égard aux droits du texte, l'intentio operis ; modèle que nous avons élargi à la peinture comme J.C. Chabanne et J.-L. Dufays aux autres arts (2011). Nous avons défini des caractéristiques langagières qui nous ont permis d'établir des typologies des écrits des élèves selon qu'ils s'approchaient plus ou moins de ce modèle ${ }^{3}$.

4 Nos résultats montrent qu'ils en sont majoritairement plus proches sur la peinture que sur la littérature et que cette différence s'accroit dans les établissements de recrutement plus défavorisé. Dans la recherche globale, nous en proposons pour explication les écarts sémiotiques entre les deux arts, qui ont nécessairement des incidences herméneutiques (notamment Marin, 1972), et, par conséquent, didactiques ${ }^{4}$ : ce qui nous amène à la conclusion que le transfert des compétences acquises d'un exercice à l'autre ne peut pas être immédiat et que sa réussite est subordonnée à un étayage spécifique de la part des enseignants, qui consiste à amener les élèves à identifier ce qu'ils réussissent dans un exercice pour le transposer à l'autre. Pour autant, à la condition que soit prise en charge cette médiation du retour au bénéfice de la lecture littéraire des apprentissages réalisés à partir de la peinture, le détour peut être une ressource précieuse à mobiliser en classe. Pour y parvenir, il est cependant nécessaire de tenir compte du second facteur explicatif que nous faisons apparaitre de la meilleure réussite sur la peinture et qui concerne le rapport différent qu'ont les élèves aux deux arts: souvent peu préparés par leur expérience extrascolaire à la réception de la peinture, notamment religieuse, manquant de ce fait souvent des savoirs culturels qu'elle réclame, ils déclarent pourtant très majoritairement en entretien en avoir plus de gout et de familiarité que de la littérature.

C'est l'examen de cet apparent paradoxe que nous nous proposons d'approfondir par cet article : le fait que les élèves ne s'investissent pas de la même façon dans la réception de 
l'un et l'autre des deux objets, qu'ils ne leur donnent pas la même place dans leurs préférences culturelles, nous semble une donnée essentielle à analyser et à prendre en compte pour la réussite du détour et de son retour.

\section{Cadre théorique}

6 Même si nous nous appuierons sur des éléments issus des sciences de l'art et de la didactique du littéraire, nous mobiliserons ici prioritairement les cadres sociologiques de notre recherche, qui sont ceux de la sociologie de la culture (Bourdieu \& Darbel, 1966) et de la sociologie scolaire, plus précisément ce qui, dans l'approche curriculaire, interroge la façon dont la forme scolaire met à l'épreuve les dispositions (Bourdieu \& Passeron, 1970) dont les acteurs sont différemment porteurs (notamment Bautier \& Rayou, 2013).

7 Notre approche, même si elle se réfèrera à certains résultats quantitatifs issus de notre recherche doctorale, sera principalement qualitative et tentera d'accéder au rapport que les élèves ont aux deux objets, c'est-à-dire à leurs logiques d'analyse. Les registres cognitif, culturel et identitaire symbolique, tels que les définissent P. Rayou et É. Bautier ( ibid.), nous fourniront un outil d'analyse puis de comparaison (d'un contexte social à l'autre et d'un art à l'autre) de ces logiques telles qu'elles apparaissent dans les textes des élèves et dans les entretiens postpassation: selon ces auteurs, "les apprentissages scolaires mettent en jeu des registres différents et complémentaires de mobilisation des élèves" (ibid., p. 32). Le premier est "cognitif», constitué, pour l'activité qui nous intéresse, d'opérations permettant ou non, selon les cas, de construire et d'étayer une interprétation solide et cohérente. Le deuxième registre, « culturel », est fait, dans notre cas, de savoirs linguistiques, iconographiques, ou encore de savoirs généraux sur les contextes de production et de réception. Le troisième est "symbolique »: une activité scolaire «construit un certain type d'identité personnelle et relie à une communauté pour laquelle il vaut » (p.32); dans le cadre du processus dialectique de construction du sens tel que nous l'avons défini, il faut accepter d'investir son émotion et ses valeurs dans sa réception mais aussi de justifier ses hypothèses en fonction des caractéristiques objectives du texte ou du tableau, car c'est nécessaire pour les communiquer à autrui ; ce processus d'investissement de soi puis de ressaisie des fruits de cet investissement n'est pas anodin sur le plan identitaire. Ces registres nous semblent donc pouvoir fournir aux enseignants un outil d'analyse fine du mode d'appropriation par les élèves de l'un et l'autre des deux objets, et de ce fait une aide pour l'étayage du transfert des apprentissages d'un art à l'autre, dans le cadre d'une pratique du détour par la peinture. Nous y reviendrons plus précisément à partir d'exemples de textes d'élèves. Mais nous commencerons par l'acceptation plus fréquente de l'exercice proposé sur la peinture, ce qui est un premier élément de connaissance du registre que nous nommons de l'identité symbolique.

\section{L'impression de familiarité et de compétence}

\section{Le choix moins fréquent de ne pas traiter la peinture}

Une partie des élèves de notre corpus a choisi de ne pas traiter l'un des deux objets, comme le proposait la consigne. Ils sont sensiblement plus nombreux à ne pas traiter le texte $(30,5 \%)$ que la peinture $(5,5 \%)$. L'écart est plus net encore si nous ne retenons que 
les travaux des élèves scolarisés dans des collèges de recrutement majoritairement défavorisé ( $47,5 \%$ laissent de côté le texte et $1,5 \%$ la peinture). Or nous avions constitué notre corpus de manière à harmoniser autant que possible le niveau de difficulté entre peinture et littérature (même si rien, bien sûr, ne peut garantir indiscutablement cette équivalence). Pour chaque corpus, la proximité temporelle des œuvres devait favoriser une certaine homogénéité des implicites culturels nécessaires à leur appréhension. Plus précisément, pour le corpus du XX $\mathrm{XX}^{\mathrm{e}}$ siècle, un extrait d'Orphée de J. Cocteau (1927) et La Crucifixion blanche de M. Chagall (1938) impliquaient l'un et l'autre des références culturelles savantes. Un extrait de Sido de Colette (1929) soulevait la difficulté d'une œuvre intimiste, comme c'était le cas aussi de La famille de saltimbanques de P. Picasso (1905). Enfin, au caractère très musical du « Pont Mirabeau » de G. Apollinaire (1913) nous semblait pouvoir faire pendant Paysage sous la pluie de V. Kandinsky (1913), tableau exigeant la prise en compte des effets de sens des teintes, des formes et des textures. Pour le corpus $\mathrm{du} \mathrm{xVII}^{\mathrm{e}}$ siècle, nous avions recherché le même type d'homogénéité en proposant Stances à Marquise de P. Corneille (1658), la scène 1 de l'acte V d'Andromaque (1667) de J. Racine et deux tableaux dont la difficulté d'accès nous semblait valoir celle que pouvait poser aux élèves la langue du XvII ${ }^{\mathrm{e}}$ siècle : La Nativité ou le Nouveau-né de G. de la Tour (entre 1645 et 1650), scène traitée de façon peu conventionnelle; et Ulysse remet Chryséis à son père de C. Gellée (vers 1644), dont la référence à L'Iliade présente dans le titre est très peu explicative du tableau et dont le traitement des figures est allusif et peu narratif. Enfin Le Tricheur à l'as de carreau de G. de la Tour (vers 1635), scène de genre très théâtralisée, nous paraissait présenter le même type d'intérêt et de difficulté que la scène 10 de l'acte III du Malade imaginaire de Molière (1673). La passation étant indépendante de toute séquence didactique et de toute évaluation, le fait que bien plus d'élèves choisissent de ne pas traiter l'extrait littéraire nous semble donc pouvoir s'expliquer par l'impression d'une meilleure compétence sur la peinture, et non par une facilitation effective induite à priori par un objet ou par l'autre.

\section{La déclaration de préférence}

9 D'après nos entretiens postpassation, les élèves que nous avons interrogés disent ne pas donner aux deux arts la même place dans leurs préférences culturelles. Ils se déclarent plus réceptifs à la peinture, qui les émeut, alors que le texte les laisserait de marbre, comme le montre cet extrait d'entretien, choisi parmi de multiples autres exemples :

Hafza : Les émotions du texte moi je les sens pas ! [...]

$\mathrm{C}^{5}$ : Et sur les tableaux ça vous fait quelque chose davantage?

Sarah: Oui parce que il y a des couleurs.

Hafza : Et c'est un dessin on peut voir quand c'est sombre, que c'est obscur, qu'il veut exprimer la tristesse par exemple... alors que si on me donne un texte je vais pas être triste [...].

$\mathrm{C}$ : Il y a quand même déjà des textes qui t'ont donné des émotions comme un tableau?

Sarah : Non [...] jamais.

10 Si nous comprenons cet échange eu égard à J.-M. Schaeffer (2015) qui décrit l'expérience esthétique comme attentionnelle, émotive et hédonique, parmi ces trois ressorts l'émotion serait donc, pour ces élèves, activée par la peinture plus aisément que par la littérature. 
11 Outre que la peinture les touche davantage, le musée d'art, « univers sacré de la culture légitime » (Bourdieu \& Darbel, 1966, p. 89), est plusieurs fois décrit comme un lieu désirable. Ainsi, lors d'un entretien enregistré au musée du Louvre $^{6}$ avec Karim et Mohamed, ce dernier vient de déclarer qu'il aimait ce musée car y venir « Ça fait le mec intelligent... Ça fait le mec qui sait des choses sur la vie [...] le mec cultivé ». Nous leur demandons si la lecture de la littérature leur fait le même effet :

Mohamed : Non non, avec les textes c'est pas pareil, c'est pour l'école...

C. : Mais ça fait pas cultivé les textes ?

Mohamed : ... Non ben pas tellement...

Karim : La culture c'est pas les textes...

Mohamed : Ouais on pourra dire ça à nos enfants quoi, qu'on connaît le Louvre !

C. : Tu amèneras tes enfants au Louvre?

Mohamed : Je les amène tout de suite ! Tout petit mon fils je l'amène...

12 Cet exemple est significatif de la « publicisation » d'une préférence culturelle, qui permet de se définir "une identité pour autrui » (Octobre, 2006, p. 159). Le musée est souvent revendiqué par les élèves comme un univers où ils se sentent à même de s'intégrer (Nadia au Louvre : «Il y a des endroits où on peut se sentir pas dans notre contexte mais là, non ça va... Il y a d'autres lieux où on peut se sentir en trop, mais là non »; Karim : «j'aurais pu rester trois heures de plus! ça me fait plaisir de voir tout ça !»). Le ressort hédonique de J.-M. Schaeffer serait donc activé lui aussi.

Qu'en est-il de l'attention, que J.-M. Schaeffer décrit, en «mode esthétique» (p.12) comme plus fine et finalisée de façon plus ouverte que l'attention standard? Les élèves que nous avons enquêtés disent que le tableau leur serait plus ouvert que le texte. En effet, il est " déjà écrit » (Amélie : « sur le tableau il y a rien d'écrit, c'est à nous d'écrire, alors que sur le texte il y a ce qu'il y a déjà écrit »). Il leur permettrait de participer à la conception du sens alors que le sens du texte littéraire se réduirait forcément aux intentions de l'auteur (Dayane : «On interprète par rapport à ce que nous on pense... alors qu'un texte on essaie de trouver ce que l'auteur... il a voulu nous faire montrer... »). La peinture s'ouvrirait à des interprétations multiples alors que «pour la plupart des textes il y a qu'une seule manière de le comprendre" (Hafza). Pour J.-M. Schaeffer l'attention esthétique est aussi plus informée, traitant en parallèle «plusieurs sources informationnelles différentes »(p.317). Les élèves se prévalent justement lors de nos entretiens de disposer de compétences et de savoirs qui le leur permettraient: " on est sûr, on ne peut pas se tromper parce que c'est ce que nous on voit...» (Akim). Certes plusieurs sens sont possibles, précise-t-il, mais en tout cas on est « hors de la tromperie ». Plusieurs n'en restent pas à une aisance présumée comme naturelle et se déclarent capables de mobiliser ce qu'ils savent pour appréhender la peinture. Akim explique : «Déjà juste quand on sait un peu la vie on sait... on peut comprendre... on regarde un tableau, on sait... l'atmosphère... [...] si c'est une atmosphère chaleureuse ou intrigante... » Donc selon lui le bagage culturel dont il dispose lui permet de comprendre la peinture, non seulement de reconnaitre ce qui est figuré, mais au-delà d'appréhender ce qui caractérise la vision du peintre, l'atmosphère qui s'en dégage. D'autres entretiens manifestent cependant la conscience que des savoirs issus de la culture légitime y sont utiles aussi (Karim : « il faut connaitre l'histoire, voir des liens quoi... sans culture on peut pas comprendre... »). Mais ceci n'empêche pas qu'ils se sentent capables de produire une analyse pertinente d'une peinture : Selim et Dayane nous expliquent que contrairement à ce qui se passe pour un texte, un tableau les amène à «faire le rapport [...] à nos apprentissages d'avant [...] la culture générale... à l'école on apprend des choses alors 
peut-être ça va nous donner le déclic par rapport au tableau... » Selim précise à propos de La Nativité de G. de la Tour : « on a déjà vu des tableaux avec de la lumière qui met en valeur les choses de Dieu...». Dayane ajoute aux savoirs interpicturaux les savoirs contextuels : «Je serais pas au courant de la naissance de Jésus je pourrais pas inventer quelque chose...»

Ces quelques exemples sont représentatifs des réponses que nous avons obtenues, dans lesquelles les élèves revendiquent fréquemment du gout et de la compétence pour la peinture, qu'ils déclarent accueillir bien volontiers dans leurs préférences culturelles, alors qu'ils désignent de façon récurrente la littérature comme un objet purement scolaire pour lequel ils se sentent en moindre familiarité.

\section{L'impression de familiarité et de compétence : illusion ou vérité ?}

\section{Une part d'illusion}

D'abord, cette impression est en contradiction avec les enquêtes sociologiques sur les pratiques culturelles. O. Donnat (2011) constate grâce à une analyse rétrospective des résultats de cinq enquêtes sur les pratiques culturelles des Français entre 1973 et 2008 «la permanence d'une forte stratification sociale des pratiques culturelles» (p. 28). Notamment, la visite au musée reste une pratique de distinction. Pour ce qui est plus précisément du jeune public, l'enquête menée en 2004 pour le ministère de la Culture par Sylvie Octobre (2006, p. 154) montre que la fréquentation des « lieux de patrimoine et de spectacle" se fait surtout, pour ce qui est du musée, dans le cadre scolaire; elle ne concerne que $50 \%$ des enquêtés et chute de $13 \%$ de la maternelle à la troisième, alors que la lecture est pratiquée par $94 \%$ des enquêtés. La différence de la fréquentation des lieux de patrimoine et de spectacle entre enfants d'ouvrier qualifié et enfants de cadre est de 22,5 points alors qu'elle n'est que de 18 points pour ce qui concerne la lecture quotidienne (Octobre, 2008, p. 32). La visite au musée hors du cadre scolaire reste donc la pratique de distinction que montrait l'enquête de P. Bourdieu et A. Darbel en 1966 dans L'amour de l'art : « croissant fortement à mesure que le niveau d'instruction s'élève, la fréquentation des musées est presque exclusivement le fait des classes cultivées » (p. 35).

Ensuite, notre analyse des textes des élèves montre que les déficits culturels mettent en réalité plus souvent les élèves en difficulté quand ils travaillent sur la peinture que quand ils travaillent sur la littérature. Ainsi repérons-nous des défaillances culturelles que nous montrons comme interdisant la prise en compte des droits de l'œuvre dans $43 \%$ des textes sur la peinture et dans seulement $27,5 \%$ des textes sur la littérature. Comment ceci peut-il s'expliquer?

17 Pour ce qui est de la compréhension du texte, différentes « encyclopédies " sont définies par U. Eco (1985, p. 68) comme nécessaires au lecteur modèle, c'est-à-dire celui qui respecte les droits du texte: savoirs linguistiques, contextuels (contexte diégétique ou contexte de production de l'œuvre), artistiques (connaissances génériques, historiques, intertextuelles...). Ces encyclopédies nous ont semblé pouvoir être transposées dans le cadre scolaire. Par exemple, quand Tarik écrit à propos du poème de G. Apollinaire : « Le "Pont Mirabeau" se trouvant à Paris, je ne comprends pas pourquoi Vienne est citée, surement pour que ce soit poétique», nous avons considéré qu'il y avait un déficit du 
savoir linguistique mais aussi contextuel (même s'il sait que le contexte est parisien, Tarik ne parvient pas à rectifier son erreur par l'appui sur ce savoir) et artistique, puisqu'il se réfère à une connaissance peu satisfaisante du genre poétique, qui conforte son erreur linguistique. Pour ce qui est de la peinture, s'il n'existe pas de « lexique visuel », elle exige des connaissances iconographiques, parfois savantes : elles permettent au spectateur de passer du niveau pré-iconographique, défini par E. Panofsky $(1967$, p. 17, 18) comme la reconnaissance de ce qui est figuré, c'est-à-dire les "motifs artistiques» (notre " expérience pratique » nous suffit à les identifier et à en identifier la valeur expressive ${ }^{7}$ ) au niveau iconographique, par lequel le spectateur accède aux significations conventionnelles et reconnait des « thèmes » ou " concepts » (la Nativité, la Crucifixion... ), dont le traitement pictural est plus ou moins fidèle à la tradition, les écarts ayant du sens. Bien d'autres encyclopédies sont souvent nécessaires pour respecter les droits du tableau, et peuvent mettre les élèves en difficulté. Ainsi de Cynthia, qui écrit sur la Crucifixion blanche de M. Chagall : « on aurait dit qu'il y a la guerre, tous le monde fuit et y a un seul homme sur un poto, des maisons brulent, des personnes dans les rues ou dispersées ». Le contexte de production est ignoré alors que la date de l'œuvre, 1938, l'année de la Nuit de Cristal, est exploitée par d'autres élèves (par exemple Mohamed: "Mais avec la date dans le titre on peut interpréter ce tableau d'une autre manière qui est liée avec l'avant seconde guerre mondiale »). Cynthia ne reconnait pas le double contexte diégétique, chrétien et juif (par opposition, Marc écrit : « nous retrouvons la Thora dans le coin droit en bas de l'image ; une synagogue au-dessus est en flamme. Nous voyons sous le corps du Christ, le bougeoire a 7 branches»), double contexte qui permet de comprendre l'universalisation de la souffrance du Christ, élargie à celle du peuple juif persécuté. Enfin et surtout, Cynthia ne reconnait pas le thème de la crucifixion. Au contraire, quand Naomé écrit : «Les anges ne sont pas représenter sous forme de vrais anges [...] seul Jesus est représenter sous sa vrai forme d'être saint", elle conjugue reconnaissance du thème et connaissance interpicturale, appréhendant le tableau dans ses échos et écarts avec la tradition iconographique chrétienne, ce qui pourra lui permettre d'accéder au renouvellement de sens conféré au thème de la crucifixion. Contrairement à Cynthia, elle se montre capable de percevoir la représentation comme pouvant recevoir des interprétations symboliques plurielles, appuyées ou non sur la convention iconographique, et pas seulement comme restitution d'un visible réel.

La proximité culturelle avec la peinture n'est donc pas confirmée. Doit-on pour autant considérer comme illusoire et vaine l'impression de compétence que les élèves expriment fortement à propos de la peinture ? Il nous semble que non, pour plusieurs raisons.

\section{L'impression de familiarité et de compétence : une part de confirmation}

\section{Des réquisits culturels hétérogènes selon les tableaux}

19 Les résultats obtenus à l'analyse du registre culturel sont très hétérogènes, les trois tableaux qui dans notre corpus sont concernés par le recours à une iconographie conventionnelle concentrant les difficultés: seuls trois élèves ne reconnaissent pas la Crucifixion dans le tableau de M. Chagall, mais $64 \%$ ne saisissent pas la référence au judaïsme ; 66,5\% des élèves n'identifient pas le costume d'Arlequin, ni plus largement le topic du théâtre, pour La famille de saltimbanques de P. Picasso; enfin $74 \%$ des élèves qui ont travaillé sur La Nativité ou le Nouveau-né de G. de la Tour ne reconnaissent pas la Sainte 
Famille. Les autres tableaux ne suscitent pas plus de difficultés que les textes. D'ailleurs, dans les collèges de recrutement défavorisé, le registre culturel devient plus favorable à la peinture, notamment parce que les difficultés face aux textes littéraires sont plus fréquentes, le taux des travaux sans défaillances culturelles majeures chutant de presque 20 points ; mais aussi, de façon plus surprenante, parce que le taux de textes dans le même cas sur la peinture augmente un peu, ce qui s'explique notamment par de moindres difficultés à reconnaitre l'iconographie religieuse dans La Crucifixion blanche et dans $L a$ Nativité ou le Nouveau-né. La même différence ne nous apparait pas pour le texte de $\mathrm{J}$. Cocteau et le tableau de C. Gellée à propos des mythes gréco-romain. Globalement, le registre culturel met les élèves des collèges de recrutement défavorisé bien plus en difficulté que les autres par rapport à la littérature, alors que pour la peinture l'écart est moindre. Vraisemblablement, les pratiques familiales marquent moins la différence d'un contexte social à l'autre pour ce qui concerne la peinture que pour ce qui concerne la littérature. Ceci peut signifier que les résultats de P. Bourdieu et A. Darbel restent d'actualité : la peinture n'est vraiment familière qu'aux milieux très cultivés, visiblement peu représentés dans notre corpus, même dans les établissements de recrutement plus favorisé. D'autres facteurs nous semblent cependant pouvoir expliquer cette différence entre les deux arts.

\section{Les autres registres au secours du registre culturel : l'exemple de Célia}

20 Les registres tels que nous les définissons interfèrent, de sorte qu'il est nécessaire de les considérer dans leur complémentarité pour comprendre l'activité mise en œuvre par les élèves. Or les deux autres registres nous apparaissent comme plus favorables à la peinture, l'écart d'un art à l'autre se creusant dans les collèges de recrutement défavorisé. Pour ce qui est du registre cognitif, les élèves envisagent plus souvent plusieurs possibilités de sens, ils descendent à un niveau d'attention plus fin aux détails, ils prennent plus volontiers en compte, en lien avec les signes iconiques, les signes plastiques (système des teintes, textures, graphisme, composition...). Pour ce qui est du registre symbolique, il concerne l'engagement du sujet dans la construction du sens : dans le processus dialectique que nous avons décrit, l'approche est nourrie des émotions et des valeurs du lecteur, qui fournissent un matériau à retravailler, à ressaisir à la lumière de l'analyse précise de l'œuvre et de références culturelles légitimes, de manière à concilier les droits du récepteur avec les droits de l'objet. Pour l'analyser dans les textes des élèves, nous avons défini des marques langagières caractéristiques de l'investissement subjectif, d'autres marques caractéristiques de sa ressaisie, enfin des marques du désengagement du sujet (voir notamment Claude, 2016a).

21 Le texte de Célia sur La Nativité ou le Nouveau-né de G. de la Tour nous semble permettre d'illustrer, à condition d'une analyse fine, l'interaction des deux autres registres avec le registre culturel :

[...] Le rouge représente le sang, la maman qui a souffert pour accoucher, tous ceux qu'elle va devoir faire pour lui, la main à droite du tableau (celle de la maman) est représentée avec des traits, des gravures, ceci représente la fatigue. A cause du noir, on ne voit que les trois personnages, ceci veut dire que la maman ne pense qu'à son bébé, ne voit que celui-ci, tout ce qui compte pour elle est le bébé. Le bébé est représenté avec une lumière blanche, donc c'est un espoir, une sorte d'ange pour la maman, une étoile, ça met en valeur à quel point celui-ci est important aux yeux de sa maman. La dame à gauche est beaucoup plus vieille que la dame en rouge. Donc je conclus que ça peut être la mère de la dame en rouge, qui est 
toujours là pour soutenir sa fille pendant un moment si important. Pendant des années elle a fait ça et elle continue. Ici on voit la représentation de 3 générations : la grande-mère, la mère et le bébé. La vieille dame a un vêtement de couleur maron et blanc, le marron représente pour moi le sang rouge qui a séché, donc les années, alors que la jeune dame est encore toute fraiche avec la couleur rouge [...]. Pour conclure, le peintre montre l'importance des mères, ceux qu'elles peuvent faire pour leurs enfants, à quel point elles souffrent.

Pour le registre culturel, la connaissance iconographique fait défaut: comme nombre d'élèves, Célia ne reconnait pas les personnages évangéliques et ignore sans doute le sens du terme Nativité dans le titre. Elle voit la scène comme une scène familiale intime, un nouveau-né entouré de sa mère et de sa grand-mère. Son investissement des deux autres registres lui permet pourtant de construire une interprétation à partir de cette compréhension qu'on peut considérer comme partiellement défaillante. Le sujet de l'énonciation est visible par les pronoms, les modalisateurs et les verbes explicitant son activité (ceci veut dire, ça met en valeur à quel point, etc.). Mais nous ne considérons pas ces marques comme une preuve suffisante de la constitution du récepteur en instance sémiotique si elles ne sont pas confirmées, sur le plan sémantique, par ce que nous nommons un décrochage entre le descriptif, l'énoncé de ce qui immédiatement et incontestablement attaché au tableau et les effets de sens interprétés. Ce décrochage est notamment marqué par le passage de l'observation descriptive (des traits, des gravures, une lumière blanche, le marron, la couleur rouge, etc.) à l'abstraction (la fatigue, l'espoir, les années qui passent, etc.); ou du singulier au général: cette famille comme symbole de la succession des générations, les deux femmes comme une allégorie de la maternité. Ces hypothèses nous semblent justifiées, du moins partiellement: Célia s'appuie sur les caractéristiques iconiques (le dévouement entier de la mère à l'enfant visible à la position des personnages, qui forment bloc, et à leur expression) et plastiques (sens symbolique du rouge, jeu sur le contraste avec le marron, perçu comme une altération du rouge, interprété comme la continuité de la douleur d'une génération à l'autre; contraste entre le fond sombre et la lumière qui met en valeur l'enfant consolateur ; composition en plan serré sur les trois personnages, qui exclut toute diversion anecdotique). Célia produit ainsi un pan profane de l'interprétation, qui serait pertinent pour la construction d'un sens pluriel, l'interprétation religieuse n'en étant pas exclusive, bien au contraire : ce tableau s'attache à représenter la Nativité comme une naissance, Jésus comme un bébé ordinaire, excluant les indices conventionnels les plus spectaculaires (ni aura, ni envolée d'angelots, ni bœuf ni âne...) pour mettre l'accent sur le mystère de l'incarnation. Le processus de ressaisie est donc en cours dans le travail de Célia, mais il y manque le savoir iconographique et la connaissance de ses significations religieuses. Ce savoir pourrait lui permettre de réinterpréter ce qu'elle perçoit pour construire la polysémie: l'enfant messie comme lumière et espoir, non d'une famille seulement, mais de l'humanité ; la proximité plastique de Sainte Anne et de Marie comme transposition picturale du dogme de l'Immaculée Conception; la prescience de la Passion dans l'expression presque douloureuse de Marie... Ce complément serait nécessaire pour que le tableau soit respecté dans tous ses droits. Mais on peut considérer que l'activité cognitive de Célia, sous-tendue par un engagement subjectif actif, mais aussi par son bagage culturel personnel (les topics du sang de l'accouchement comme symbolique de la condition féminine ou la figure de Mère Courage sont certes anachroniques, mais ils nourrissent l'interprétation), lui a permis de produire le premier jet d'une interprétation qu'un enseignant pourrait l'aider à réécrire par un étayage culturel. 


\section{L'impression de familiarité : un moteur pour l'investissement de soi}

La peinture génère plus souvent que la littérature des textes qui nous paraissent présenter, comme dans le cas de Célia, les marques d'un engagement du sujet dans le travail de construction du sens ( $86 \%$ du corpus global sur la peinture contre $72,5 \%$ sur la littérature) : la familiarité culturelle ressentie par les élèves, même si elle n'est pas effective, les amène à s'autoriser davantage à jouer le jeu. Dans le cadre d'une lecture littéraire conçue comme une dialectique entre investissement et ressaisie, cet investissement est très important, «l'essentiel étant de faire quitter aux élèves cette posture d'extériorité construite face à un objet scolaire pour les amener à comprendre que l'œuvre s'adresse à eux » (Rouxel, 2007, p. 72).

4 S. Octobre souligne que, pour le sociologue, comprendre les pratiques culturelles des adolescents nécessite de croiser les informations objectives (fréquences de consommation ou de pratique) et subjectives (gouts déclarés) car ils peuvent ne pas valoriser ce qu'ils pratiquent beaucoup et valoriser beaucoup ce qu'ils pratiquent peu : «la vision de leur rapport avec les loisirs culturels est tout autre dès lors que l'on déplace l'observation de ce qu'ils font à ce qu'ils déclarent aimer faire» (2006, p.154). Notre corpus permet d'appréhender une incidence didactique de ce décalage : même si leur pratique du musée et de la réception de la peinture est sans doute moins fréquente que leur lecture de la littérature, même s'ils ne disposent pas plus souvent des références légitimes, la familiarité dont ils se réclament et le sentiment d'y être compétent permettent une meilleure acceptation de l'exercice, un engagement subjectif plus entier, ce qui est favorable au processus de construction du sens (le texte produit sans étayage culturel doit être considéré comme un état provisoire qu'il faudra les aider à ressaisir).

\section{Culture juvénile et investissement différencié du sujet d'un art à l'autre}

Si le travail sur la littérature génère plus souvent que la peinture le désengagement subjectif (selon nos analyses, $54 \%$ des cas contre $39 \%$ ), il est loin d'être général. Mais cet investissement subjectif n'est pas forcément de même nature et il est plus souvent insuffisamment ressaisi ( $15 \%$ des cas contre $8,5 \%$ pour le corpus global et $31,5 \%$ contre $16,5 \%$ dans les collèges de recrutement plus défavorisé).

\section{L'investissement axiologique : plus fréquent face au texte littéraire}

Il arrive souvent qu'un personnage soit perçu non comme une construction du texte, possiblement signifiante au même titre que ses autres composantes, mais comme une personne réelle dont les comportements heurtent les valeurs du récepteur (on rejoint le texte action de la typologie de Bucheton, 1999). Ainsi Oriol écrit-il à propos de l'extrait d' Orphée: « Mais le personnage il se prend trop pour un homme qui va être important dans le monde, il a cru qu'avec sa phrase "Eurydice reviendra des enfers", il allait venir le premier poète le plus connu dans tout le monde ». Si Oriol perçoit ainsi le personnage d'Orphée, c'est parce que le texte le traite de façon burlesque et parodique : persuadé de la qualité poétique d'un vers dont il ne comprend pas qu'il est un acrostiche (Madame $\mathbf{E}$ urydice Reviendra Des Enfers), le personnage se prend effectivement pour le grand poète 
qu'il n'est pas. Le jugement dépréciatif pourrait donc nourrir le processus interprétatif, si Oriol interrogeait le texte pour savoir ce qui a suscité sa réaction. Au lieu de quoi il met fin à sa rencontre avec le texte - ou avec le personnage : « sa me donne pas envie de lire l'histoire ». Le niveau de langue très familier, affecté des marques de l'oralité (il se prend trop, il a cru, en plus, sa me donne pas envie), alors même que l'orthographe presque correcte, laisse penser qu'il serait capable d'écrire de façon plus scolaire, peut être interprété comme une façon de manifester sa fidélité à sa culture et ses valeurs personnelles, au nom desquelles il ne saurait accepter de s'intéresser à un personnage qui manque à ce point d'honnêteté sur lui-même. Valeur qui nous semble ancrée dans la culture juvénile puisque nous la retrouvons plusieurs fois à propos d'objets très différents. Par exemple Mustapha laisse toute la place à l'expression sincère de sa désapprobation concernant l'extrait de Colette, qui dit «que du bien » du personnage et non "la vériter car tout le monde c'est quand des qualités et des défault et non que des qualités ». Nous retrouvons plusieurs fois ce même appel à la modestie à propos du poète de Stances à Marquise déclarant à la jeune femme : "Vous ne passerez pour belle / Qu'autant que je l'aurai dit ». Dans tous les cas, le jugement de valeur, tout maladroit qu'il soit, pourrait être ressaisi à la lumière d'un savoir culturel qu'apporterait un enseignant pour redonner place aux droits du texte et du contexte : comme moyen de la parodie, comme topos du carpe diem, comme questionnement de la reconstruction de soi dans l'écriture autobiographique.

L'engagement axiologique du sujet empirique peut aussi consister en l'expression de positions éthiques générales : qu'on retrouve souvent les mêmes à propos d'œuvres très différentes montre qu'elles relèvent de valeurs juvéniles collectives, qui s'expriment parfois, mais pas toujours, dans l'ignorance des droits de l'objet (dans la typologie de D. Bucheton, il s'agirait alors de la posture du texte tremplin). Aussi bien La famille de saltimbanques que La Crucifixion blanche permettraient de comprendre qu'il est injuste qu'il existe des pauvres et des riches, l'extrait de Colette aussi bien que le tableau de G. de la Tour montreraient l'importance du respect de la famille, des œuvres aussi variées que les tableaux de P. Picasso et de V. Kandinsky et que l'extrait de Colette seraient au service d'un plaidoyer écologique. Le texte d'Elia sur l'extrait de Colette illustre bien ce dernier exemple : «comme quoi la nature est vraisemblablement la plus belle chose qui puisse exister sur terre. Elle est simple et jolie loin de tout comportements révoltants des humains et de la bêtise de ceux-ci ».

Ce type d'engagement est moins fréquent sur la peinture, mais n'en est pas absent. Nassima projette par exemple sur le tableau de V. Kandinsky la même préoccupation qu'Elia sur le texte de Colette : « Le peintre a fait un travail de couleur en les mélangeants comme si tout ce qui était sur terre n'était que de la peinture à l'eau qui s'éffaçait sous de l'eau. Je vois des maisons dont leur toits sont multicolore. Il $\mathrm{y}$ a une autoroute où les couleurs sont abimées. Sur ce tableau, le monde a perdu sa joie et sa beauté avec. Il n'a plus de valeur. C'est ce qu'il est en train de se passer, le monde perd tout ce qu'il a fait de lui le monde et tout ça à cause de nous : on ne sait plus apprécier sa beauté ». Comme Elia, elle fait référence à une valeur ancrée dans son ici maintenant (l'identification d'une autoroute va dans ce sens). Pourtant, cet engagement axiologique nous semble moins hégémonique car, alors qu'Elia semblait compter sur le caractère d'évidence partagée de sa conviction, Nassima cherche à justifier son interprétation par le travail pictural sur la texture et les teintes, qui rendrait visible la déliquescence du monde, dont la forme ancienne serait perceptible en filigrane (on reconnait un paysage, mais il est brouillé, abimé); quant à la responsabilité de l'homme dans cette déperdition, un enseignant 
pourrait la faire retravailler, en partant de ce qu'elle en perçoit, sur l'interprétation de mise en abyme du geste du peintre délayant et mélangeant les couleurs comme allégorie d'une action humaine dévitalisant son environnement. Donc l'engagement axiologique, tout imprégné de culture juvénile qu'il soit, nous semble en cours de ressaisie, les caractéristiques artistiques de l'objet étant prises en compte pour l'étayer.

Si nous revenons à l'ensemble du corpus, nous constatons que la peinture génère moins souvent que la lecture l'investissement axiologique : en l'absence de paroles rapportées ou de focalisation interne, l'identification n'est pas recherchée, les personnages moins souvent jugés comme s'ils étaient des personnes; en l'absence d'un discours verbal susceptible d'être perçu comme l'expression d'un message univoque, l'explicitation des contenus éthiques passe plus souvent par l'activation d'une attention esthétique telle que définie par J.-M. Schaeffer (2015), c'est-à-dire traitant selon une finalisation ouverte de stimuli fins et diversifiés.

\section{L'investissement esthétique : plus fréquent face à la peinture}

30 La sensibilité de Nassima au travail du peintre est fréquente dans le corpus global, alors que le travail de l'écrivain est très rarement évoqué. Les caractéristiques formelles du tableau sont prises en compte plus souvent que celles du texte (77\% des cas contre 50,5\% ). Certaines spécificités de la culture des élèves nous semblent pouvoir contribuer à expliquer cette différence.

D'abord, les enquêtes sur les loisirs culturels, celles d'O. Donnat notamment, montrent que les pratiques artistiques en amateur sont en plein essor dans tous les milieux sociaux : pour les arts plastiques, « la participation à la vie culturelle est devenue plus expressive" (Donnat, 2011, p.5). Ceci est légitimé, sur le plan scolaire, par les programmes des disciplines artistiques, qui donnent une place centrale aux pratiques et à la verbalisation des intentions. La sensibilité aux caractéristiques plastiques et au processus de création de l'œuvre picturale peut en être l'effet ${ }^{8}$.

Ensuite, les cultures adolescentes nous semblent nourrir l'approche de la peinture sur le plan esthétique par le truchement d'objets visuels familiers. Bob voit dans l'Arlequin du tableau de P. Picasso « Un homme [...] déguisé en Joker comme dans Batman ». Le Joker des DC Comics des années 40 est caractérisé par des yeux et de longs cheveux rouges, un visage très blanc et un sourire exagéré. Mais ses avatars plus récents, notamment dans les films d'animation, empruntent souvent au Joker des cartes à jouer son costume multicolore de bouffon, s'éloignant du modèle d'origine pour se rapprocher de l'Arlequin italien. La référence au Joker de Batman est certes hors contexte et un apport culturel sur le personnage de la commedia dell'arte doit venir la réajuster. En l'état, elle vient néanmoins étayer, pour Bob, la perception d'un univers théâtral (c'est un déguisement), ce qui lui permet d'interpréter cette scène étrange, insaisissable, comme un theatrum mundi dont la facticité est inquiétante, d'autant que cette théâtralisation est paradoxalement liée à une impression d'incommunicabilité : «Ils ont l'air perdu dans le désert mais pourtant il ne se regarde pas pour discuter où aller. C'est peut-être parce que c'est du théâtre (avec les costume) [...] On a l'impression que ce n'est pas vrai. »

Nous pourrions donner d'autres exemples, notamment empruntés à l'univers des jeux video, qui permettent de penser que leur fréquentation abondante d'objets visuels pour le loisir a un effet sur leur appréhension de la peinture. Pour autant, ces pratiques culturelles juvéniles ne font pas des élèves des experts spontanés de la peinture, et l'apport 
de savoirs légitimes est indispensable pour rendre possible la ressaisie des droits du récepteur eu égard aux droits de l'œuvre.

\section{Conclusion}

P. Bourdieu et A. Darbel (1966, p. 16), écrivaient qu'affirmer que «l'homme de la culture de l'image serait immédiatement doté de la culture nécessaire pour déchiffrer l'œuvre picturale, image entre les images", c'est réserver la peinture à une élite tout en se convaincant qu'il n'est nul besoin de médiation pour les autres car elle « enferme assez de persuasion miraculeuse pour convertir ou retenir, pour sa seule efficace, les âmes bien nées " (ibid., p. 15). Notre recherche confirme que, concernant sa scolarisation en classe de français, il serait d'une grande naïveté de se convaincre que la peinture serait en soi plus facile d'accès que la littérature sous prétexte que les élèves seraient très familiers des images, car l'appréhension de la peinture, notamment pour ce qui est de la peinture d'histoire, qui active des conventions iconographiques, met à l'épreuve des dispositions exigeantes dont les élèves ne sont pas tous porteurs à égalité.

Pourtant, de façon qui peut paraitre paradoxale, la majorité des élèves que nous avons lus et rencontrés déclarent se sentir familiers de la peinture et à même d'en construire le sens. À l'épreuve des faits, cette conviction apparait comme une illusion, car les codes culturels nécessaires leur font souvent défaut. Mais notre approche par les registres nous permet de penser que leur engagement dans l'activité, que favorise à la fois le gout, la volonté de le manifester et le sentiment d'y être compétent, s'il est pris au sérieux et s'il est pris en compte par une didactisation appropriée, peut leur permettre de produire des écrits de réception de la peinture qui peuvent constituer une première étape de la construction du sens selon le processus dialectique que nous avons décrit. Considérés comme de premiers jets, ces écrits peuvent donner lieu à une réécriture qui, nourrie d'un apport culturel par l'enseignant, sera susceptible de satisfaire aux attendus scolaires. Cet accueil favorable de la peinture dans leurs élections culturelles et le gain d'investissement qui en découle sont d'autant plus prometteurs qu'ils concernent plus nettement encore les élèves des établissements de recrutement plus populaire. Il nous semble que la connaissance des trois registres tels que nous les avons décrits et de leurs possibles configurations peut aider les enseignants, pour ce qui concerne la peinture, à s'appuyer sur ce que les élèves aiment et savent tout en leur fournissant les apports culturels qui leur manquent encore; mais il nous semble aussi que l'analyse fine des productions de leurs élèves peut favoriser l'étayage du retour, au bénéfice du texte littéraire, des apprentissages permis par la peinture : cette analyse est en effet nécessaire pour rendre visible aux élèves le processus de construction du sens qu'ils ont mis en œuvre sur la peinture et les adaptations nécessaires pour faire de même sur la littérature.

L'analyse de l'implication subjective des élèves fait en outre apparaitre une autre différence entre peinture et littérature : l'engagement de soi n'est pas toujours refusé, loin s'en faut, dans la réception du texte littéraire, et il est souvent de nature un peu différente de celui qui prévaut dans la réception de la peinture, en ce qu'il est plus souvent nourri d'éthique que d'esthétique. Or, on peut se réjouir du «tournant éthique dans l'approche et l'étude de la littérature » qui depuis les années 1990 (Michel, 2016, p. 287) marque une certaine distance avec l'approche formelle, du moins avec ses dérives formalistes (dont il n'est pas impossible de penser qu'il a pu contribuer à ce que disent les élèves que nous avons enquêtés d'un art qui laisse peu de place à leur liberté 
d'interprétation). L'investissement éthique nous apparait, d'après les textes des élèves, comme favorisé par la littérature plus que par la peinture. En ceci l'expérience de la littérature est essentielle pour leur formation, car s'il revient au lecteur ou au spectateur de co-construire le sens du texte ou du tableau, ces objets en retour contribuent à construire le sujet au travers de l'expérience qu'il en fait: «on pourrait dire que la littérature participe du souci de soi, dans la mesure où, pour reprendre les termes de Michel Foucault, elle nous constitue en agents éthiques » (ibid., p. 304)

Les modes différenciés d'engagement subjectif que suscitent les deux arts nous semblent donc complémentaires pour la formation de l'élève, à même de réagir à l'œuvre et de ressaisir ses réactions, mais aussi pour sa formation personnelle : plutôt que de préférer un art à l'autre ou de donner à l'un un rôle ancillaire pour favoriser l'accès à l'autre, il est de ce fait pertinent, de notre point de vue, de scolariser les deux arts en parallèle, dans la prise en compte de leurs spécificités, en jouant de leurs écarts et de leur complémentarité.

\section{BIBLIOGRAPHIE}

BAUTIER, É. \& RAYOU, P. (2013) [2009]. Les inégalités d'apprentissage. Programmes, pratiques et malentendus scolaires. Paris : Presses universitaires de France.

BOURDIEU, P. \& DARBEL, A. (1966). L'Amour de l'art. Les musées et leur public. Paris : Éd. de Minuit. BOURDIEU, P. \& PASSERON, J.-C. (1970). La reproduction. Éléments pour une théorie du système d'enseignement. Paris : Éd. de Minuit.

Bucheton, D. (1999). « Les postures du lecteur ». In : Demougin, P., \& Massol, J. (dirs), Lecture privée et lecture scolaire. La question de la littérature à l'école. Actes de la journée d'étude organisée à l'IUFM de Nîmes, le 11 mars 1998. Grenoble : CRDP de Grenoble, p. 137-150.

CHABANNE, J.-C. \& DUfAYS, J.-L. (coords) (2011). « Parler et écrire sur les œuvres. Une approche interdidactique des enseignements artistiques et culturels ». Repères 43.

CLAUDE, M.-S. (2015). Commenter la peinture, commenter la littérature. Présupposés, limites et perspectives d'un détour. Thèse de doctorat en sciences de l'éducation : Université Paris 8 Vincennes Saint-Denis.

CLAUDE, M.-S. (2016a). « Lire la peinture, lire le texte littéraire à l'école : une activité de même nature ? » Éducation \& Didactique 10, p. 67-76.

CLAUDE, M.-S. (2016b). « Commentaire de la peinture et commentaire de la littérature : étude comparée de l'activité des élèves ». In : Petitjean, A. (dir), Didactique du français et de la littérature. Metz : Crem, Université de Lorraine, p. 561-579.

DAMISCH, H. (1995). Traité du trait. Paris : Éd. de la Réunion des musées nationaux.

DONNAT, O (2011). «Pratiques culturelles, 1973-2008. Dynamiques générationnelles et pesanteurs sociales ». Culture études 7, p. 1-36. 
DUFAYS, J.-L., GEMENNE, L., \& LEDUR, D. (2015) [1996]. Pour une lecture littéraire. Histoire, théories, pistes pour la classe. Louvain-la-Neuve : De Boeck.

ECO, U. (1985) [1979]. Lector in fabula. La coopération interprétative dans les textes narratifs. Trad. de l'italien par M. Bouzaher. Paris : Grasset.

ECO, U. (1992) [1976]. La Production des signes. Trad. de l'italien par M. Bouzaher. Paris : Librairie générale française.

GOMBRICH, E. H. (1971) [1960]. L'Art et l'illusion. Psychologie de la représentation picturale. Trad. de l'anglais par G. Durand. Paris : Gallimard.

GOODMAN, N. (1990) [1968]. Langages de l'art. Une approche de la théorie des symboles. Trad. de l'anglais par J. Morizot. Nîmes : J. Chambon.

MARIN, L (1972). Études sémiologiques. Écritures, peintures. Paris : Klincksieck.

MICHEL, R. (2016). « Le souci de l'éthique en littérature ». In : Petitjean, A. (dir), Didactique du

français et de la littérature. Metz: Crem, Université de Lorraine, p. 285-306.

MinistÈre De L'ÉDUCATION NATIONALE (2016). Programmes d'enseignement du cycle des apprentissages fondamentaux (cycle 2), du cycle de consolidation (cycle 3) et du cycle des approfondissements (cycle 4), Bulletin officiel spécial nº 10 du 19 novembre 2015.

остовRE, S. (2006). «Les loisirs culturels des 6-14 ans ». Enfances, Familles, Générations 4, p. 146-174.

остовRE, S. (2008). «Les horizons culturels des jeunes ». Revue française de pédagogie 163, p. 27-38. En ligne : https://rfp.revues.org/940.

PANOFSKY, E. (1967) [1939]. Essais d'iconologie. Thèmes humanistes dans l'art de la Renaissance. Trad. de l'anglais par C. Herbette et B. Teyssèdre. Paris : Gallimard.

PICARD, M. (1986). La lecture comme jeu. Essai sur la littérature. Paris : Éd. de Minuit.

ROUXEL, A. (2007). « Pratiques de lecture : quelles voies pour favoriser l'expression du sujet lecteur? » Le Français aujourd'hui 2, 157, p. 65-73. En ligne : https://www.cairn.info/revue-lefrancais-aujourd-hui-2007-2-page-65.htm.

SCHAEFFER, J.-M. (2015). L'Expérience esthétique. Paris : Gallimard.

WAYSBORD-LOING, H. (2000). L'image dans l'enseignement des lettres. Rapport de l'inspection générale. Ministère de la Jeunesse, de l'Éducation nationale et de la Recherche.

\section{NOTES}

1. Ce qui en soi peut questionner car la peinture ne fait pas nécessairement image et, même quand elle est figurative, ses effets de sens ne peuvent se réduire à ceux qui tiennent à sa fonction représentative (Damisch, 1995).

2. La formulation de cette double consigne, qui se justifie par les besoins de notre recherche et non par un objectif didactique, doit être expliquée : nous souhaitions obtenir des élèves qu'ils produisent un texte rendant compte de leur réception; or notre enquête auprès des enseignants nous a permis de savoir que «donner ses impressions » était la consigne la plus fréquemment utilisée pour orienter les élèves de troisième vers ce type de texte. La consigne "commenter » était en revanche plus souvent donnée aux élèves de seconde (pour autant, l'objet de notre recherche n'est pas le commentaire en tant que genre scolaire et universitaire). 
3. Nous ne pouvons en donner le détail dans le volume de cet article: pour un aperçu synthétique, voir Claude, 2016b.

4. Ce qui peut faire douter du bien-fondé de l'expression «lecture de la peinture » dans les programmes, si elle est prise en pied de la lettre.

5. Cette lettre désigne le chercheur.

6. Nous avons réalisé certains des entretiens au Louvre afin que les élèves soient confrontés aux peintures elles-mêmes et pas seulement à des reproductions.

7. E. H. Gombrich (1971) considère qu'à ce stade déjà, la culture du spectateur est engagée, car l'artiste part de ce qu'il sait bien plus que de ce qu'il voit : l'identification par le spectateur d'un objet figuré dépend du partage de cette construction culturelle. Un seul exemple : de nombreux élèves pensent que la victime du Tricheur à l'as de carreau est une femme, car n'étant pas familiers des habitudes sociales du XVII ${ }^{\mathrm{e}}$ siècle, ils ignorent qu'un jeune homme bien né pouvait s'habiller ainsi.

8. Raison, sans doute, pour penser que scolariser davantage la pratique de l'écriture littéraire serait souhaitable.

\section{RÉSUMÉS}

Cet article s'interroge sur l'exercice que les programmes de français nomment lecture de la peinture, adaptation des exercices de lecture littéraire. D'après la recherche sur laquelle s'appuie cet article, les productions des élèves de troisième et de seconde seraient majoritairement plus proches des attendus des enseignants sur un tableau que sur un texte. La peinture, notamment celle qui en appelle à des conventions iconographiques, n'est pas en soi plus facile d'accès que la littérature et les enquêtes sociologiques montrent qu'elle n'est pas plus présente que la littérature dans les pratiques culturelles des élèves. Pourtant, la majorité de nos enquêtés déclarent se sentir plus familiers de la peinture que de la littérature et plus à même d'en construire le sens. Selon nous, ce gout déclaré, la volonté de le manifester et le sentiment d'y être compétent favorisent un engagement dans l'activité tel qu'à certaines conditions d'encadrement par l'enseignant, il est susceptible de produire des apprentissages bénéficiant à l'un et à l'autre des deux arts.

This article deals with the exercise called "image reading", in link with analytical reading of literary texts. Our research shows that the practice applied to paintings' analysis helps most students to meet academic standards. The cultural requirements of painting analysis are not less complex, especially that which resorts to iconographic conventions. Sociological surveys show that painting is not more present in students' cultural practices. Yet the majority of students surveyed say that they feel more familiar with painting than with literature and more competent for sense-building activity. According to our research, this taste for painting, the willingness to demonstrate it and the feeling of being competent in it help subjective engagement of students that under certain conditions of supervision by the teacher could produce learning process that benefits the one and the other of the two arts. 
INDEX

Keywords : art, literature, critical sense, knowledge transfert, equal opportunity

Mots-clés : peinture, littérature, construction du sens, transfert de connaissances, égalité des chances

\section{AUTEUR}

\section{MARIE-SYLVIE CLAUDE}

Université Grenoble Alpes/Centre national de la recherche scientifique, LITT\&ARTS, UMR 5316, F-38000, France 